\title{
Covid-19 orthopedic trauma patients characteristics and management during the first pandemic period: report from a single institution in Italy
}

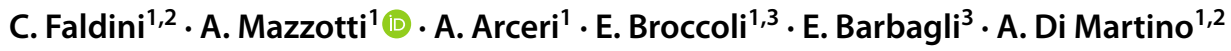

Received: 19 April 2021 / Accepted: 24 May 2021 / Published online: 2 June 2021

(c) Istituto Ortopedico Rizzoli 2021

\begin{abstract}
Purpose COVID-19 disease is a declared pandemic, affecting all aspects of healthcare, including orthopedics. The aim of this study is to describe the COVID-19 orthopedic trauma patients characteristics and management in a dedicated Orthopedic and Traumatology Hospital in Italy during the first pandemic period.

Material and methods A cohort of 25 consecutive patients with suspected or confirmed COVID-19 infection were retrospectively analyzed. Health system rearrangement, patients' clinical presentation, diagnostic tools role, laboratory finding, treatment and outcomes were evaluated.

Results Health system rearrangement was fast. There was no clear prevalence of comorbidity or surgery type between confirmed and suspected COVID-19 cases. Nine positive swabs tests and 14 cases with only suspected CT scan findings were recorded. Several laboratory changes have been reported since the onset of symptoms: anemia, leukocytosis, lymphopenia, coagulation abnormalities, alkaline phosphatase, liver enzymes and C-reactive protein alterations. Nineteen patients were treated by oxygen supplement, three patients were administered antivirals, eight antibiotic therapy, and nine hydroxychloroquine. The number of discharges reported in this study was greater than 52\% and the number of deaths reached $20 \%$.

Conclusion To our experience, the development of patient management algorithms allows the differentiation of the clinical pathways of negative and suspected/positive patients, reducing exposure, and virus spreading. The execution of swabs on all patients allows an early diagnosis and a more adequate management. Considering the different therapy patterns used, there were no significant differences, but anti-thromboembolic prophylaxis administered to all the orthopedic patients may have contributed to complications and mortality rates reduction.
\end{abstract}

Keywords COVID-19 $\cdot$ Patient management $\cdot$ Coronavirus $\cdot$ Pandemic $\cdot$ Orthopedic $\cdot$ Trauma

\section{Introduction}

The severe acute respiratory syndrome Coronavirus (SarSCoV-2 or COVID-19) has developed in China since December 2019, and quickly spread across the world, until the

A. Mazzotti

antonio.mazzotti@ior.it

1 1St Orthopaedic and Traumatologic Clinic, IRCCS Istituto Ortopedico Rizzoli, Via Giulio Cesare Pupilli 1, 40136 Bologna, Italy

2 Department of Biomedical and Neuromotor Sciences, University of Bologna, 40123 Bologna, Italy

3 Istituto Ortopedico Rizzoli Covid-Ward, IRCCS Istituto Ortopedico Rizzoli, Via Giulio Cesare Pupilli 1, 40136 Bologna, Italy
WHO declared it pandemic on March 11, 2020. China, Italy, Iran, USA, and Spain were among the most affected countries, currently overcome by the involvement of the American continent [1].

Since the beginning of the pandemic, the Italian National Health System (NHS) had to deal with several critical issues, including the need for an increase of intensive care unit (ICU) and standard hospital beds [2]. Beginning of March 2020, the regional governments decided to centralize all COVID-19 patients affected by acute diseases to selected hubs [2]. Emergency and trauma cases were centralized to dedicated hospitals, while the elective surgical activity was suspended, to support the needs of the NHS [3-5]; the rearrangement was fast, although all the changes were not simultaneous, and current protocols are the result of a continuous process of reorganization [6]. 
Emergency orthopedic patients, especially elderly subjects with fractures or tumors, have a high risk of developing complications related to hospitalization, with negative effects on functional recovery $[7,8]$. Pulmonary infections are common causes of morbidity and mortality in hospitalized patients, and the severe acute respiratory syndrome caused by COVID-19 on these patients impacts on clinical outcomes and survival [9]. Sharing patients and hospitalization characteristics, and related outcomes, may improve the management of COVID-19 infection in trauma wards, and contribute to improve the standard of care worldwide.

The aim of this study is therefore to contribute to the knowledge of the scientific community by describing the experience of a single Italian Orthopaedic center in the treatment of suspected and positive COVID-19 patients during the first pandemic period. Patient's clinical presentation, course and comorbidities, role of swab tests and CT scan, main laboratory findings, ICU access and clinical outcomes, are reported.

\section{Material and method}

\section{Study design and participants}

A retrospective study was conducted on a cohort of consecutive patients admitted between February 27 and April 28, 2020, with suspected or confirmed COVID-19 infection, at the Authors' Institution.

\section{Patients' management}

Beginning of February 2020, suspected or confirmed COVID-19 patients were managed according to the institutional guidelines done by a dedicated task force. At the admission to the Emergency Orthopedic Department all the patients were provided with a surgical mask. COVID-19 was suspected after a clinical evaluation based on epidemiological criteria including (1) history or residence in China, (2) travel in areas of known epidemic outbreak, (3) close contact to a suspected/confirmed COVID-19 patient, and (4) working/attending a healthcare facility where COVID-19 infected patients are hospitalized. Clinical symptoms including fever, cough, dyspnea and body temperature, and peripheral blood saturation values are considered. A dedicated pathway was created for suspected/positive COVID-19 patients to reduce the risk of viral contamination.

In face of a suspected case, the Public Hygiene Service took charge of the patient and gave instructions on how to proceed further, defining different pathways on the base of the risk of infection. Patients that the Public Hygiene Service considered at low risk were regularly taken in charge at the standard ward, while suspected cases underwent further diagnostic investigations, including chest X-rays, highresolution computed tomography (HRCT), swabs. Depending on tests' results, patients were placed on a COVID-19 or a standard ward. However, in case of severe respiratory distress, patients were transferred to a COVID-19 reference hospital (Fig. 1).

Patients with suspected or confirmed COVID-19 infection were managed in a separate dedicated ward, hosting all COVID-19 suspected or positive subjects, and not

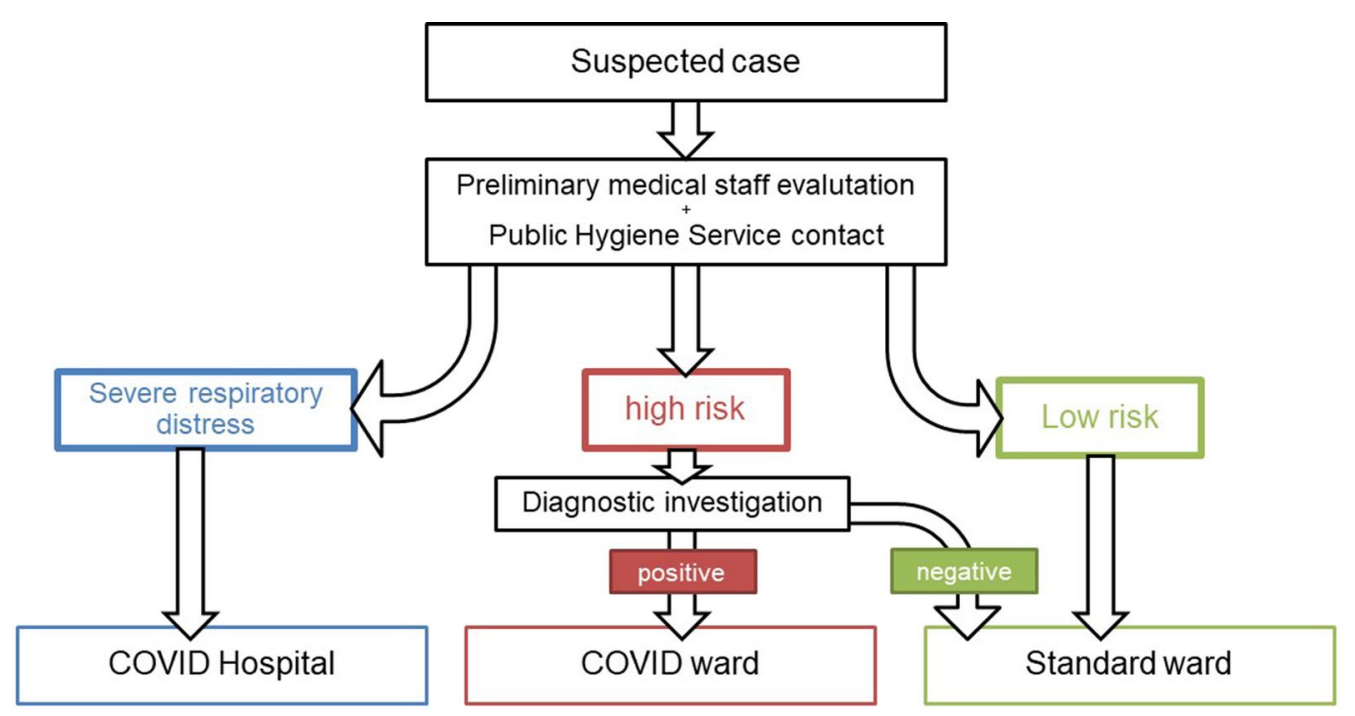

Fig. 1 Management of one suspected case 
transferred to COVID-19 hospitals because of requirement of orthopedic operation.

A separate pathway and dedicated operating theater were setup for suspected or positive patients who required surgical procedure.

For patients that developed respiratory symptoms during hospitalization, two different protocols were utilized at different times during the pandemic. During the month of March, in the event of a sudden onset of respiratory symptoms, the ward doctor evaluated the COVID-19 suspected criteria and provided hygiene measures (Table 1). Thereafter, depending on Internal Medicine and Infectiology specialist evaluations, imaging tests and NasoPharyngeal (NP) and OroPharyngeal (OF) swab for SARS-CoV-2 RNA were performed to characterize the patient as a probable or confirmed case.

Beginning of April, NP and OP swabs were performed in all patients before hospitalization, thus allowing an early diagnosis also for asymptomatic patients.

In suspected and probable patients, NP and OP swabs tests were carried out with high frequency (every one or two days), and the diagnosis of COVID-19 was excluded only upon the negative outcome of three consecutive swabs on the same patient. All the patients were considered highly suspected for COVID-19 infection if HRCT showed interstitial disease or other typical signs of viral infection (ground-glass opacities in specific areas of the lung).

All patients transferred to postoperative rehabilitation center or discharged at home received NP and OP swabs. When tested positive, the patient was placed in isolation and a transfer was planned in a healthcare facility dedicated to COVID-19 patients.

\section{Data collection}

The data considered in this study were retrospectively retrieved from patients' charts and include orthopedic diagnosis and comorbidities, signs and symptoms related to the infection, timing and modalities of the suspected COVID-19 infection and eventual evidence of COVID disease, serial laboratory tests, type of orthopedic treatment, COVID-19 infection treatment, clinical course and outcome, and characteristics of the deceased patients.

\section{Results}

\section{Patients' characteristics and comorbidities}

Twenty-five suspected or confirmed COVID-19 patients (11 males and 14 females) were included in the study. The age range was between 34 and 96 years old, with an average of 76.48. Sixteen of 25 patients were in hospital for a fracture that required surgical procedure: 14 at the femoral neck, one at the distal epiphysis of the radius, and one pathologic fracture of a lumbar vertebra. Three patients had oncological diseases: one case of chordoma, one case of paraplegia due to acute compression of the medullary canal by metastasis, and one patient affected by osteosarcoma, in-hospital for chemotherapy. The other six patients were, respectively, affected by a recurrent hip prosthesis dislocation, one surgical wound dehiscence, one lumbar disk herniation, one chronic osteomyelitis of the ankle, and one thoracic myelopathy requiring surgical decompression. All the patients had one or more comorbidities: among them the most frequent were hypertension, chronic obstructive pulmonary disease, heart disease, and malignancy (Table 2).

Table 1 Definition and therapeutic measures of suspected, probable, and confirmed cases

Definition

Initial hygiene protocols (Hand hygiene and surgical mask wearing; Droplets contact precaution; Healthcare professionals must wear appropriate $P P E$ )

Suspect case

A person with acute respiratory infection (sudden onset) and at least one of the epidemiological criteria

OR

Evidence of interstitial-alveolar pneumonia in HRCT

OR

Appearance of clinical finds ( $\mathrm{RF}>25$ acts/minute and/or $\mathrm{SO}_{2}<95 \%$ ) compatible with diagnosis of pneumonia

Probable case

Suspect case whose test result for SARS-CoV-2 is doubtful or inconclusive using specific RT-PCR protocols for SARS-CoV-2

Confirmed case

Case with a laboratory confirmation of SARS-CoV-2 infection, regardless of clinical signs and symptoms 


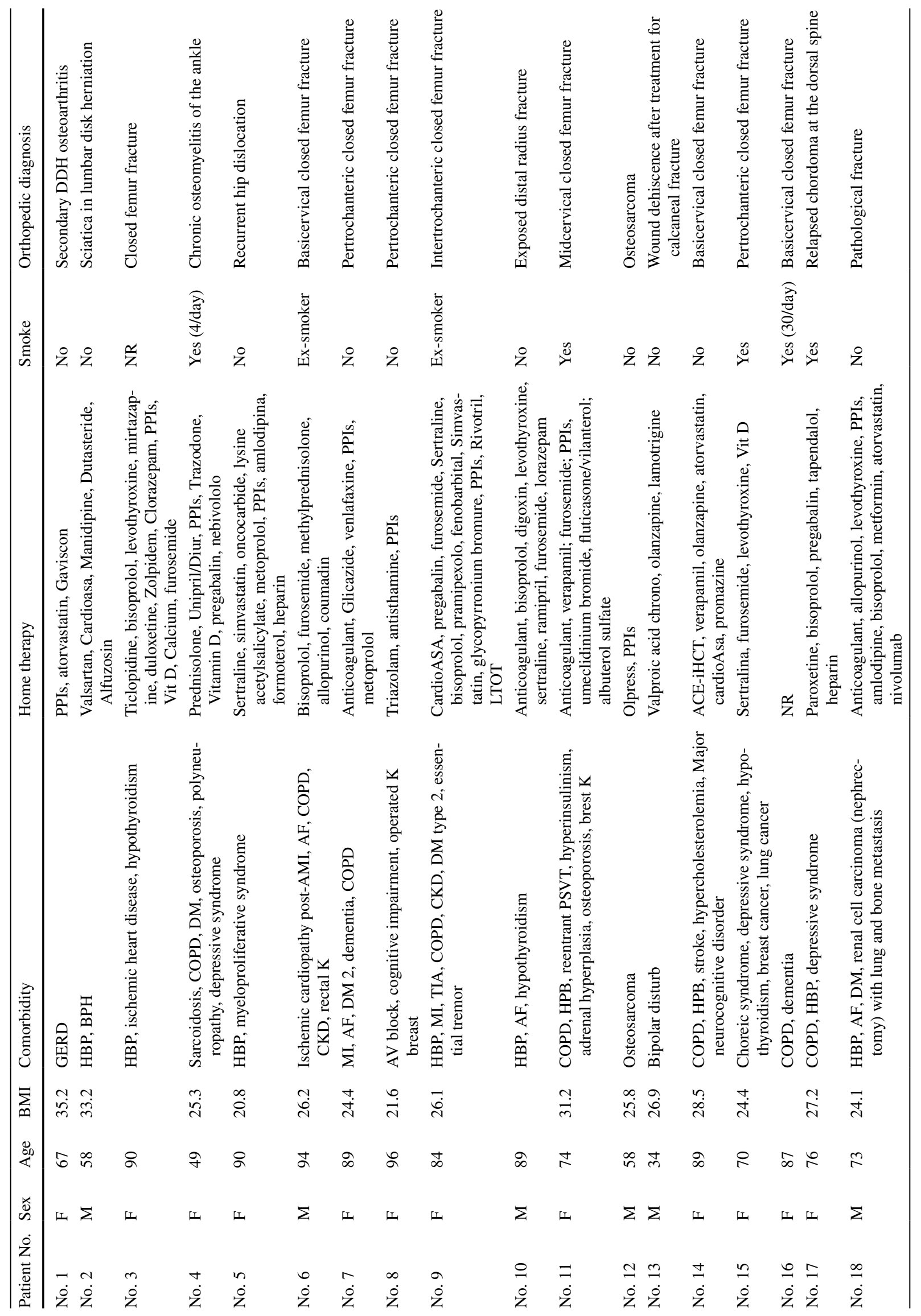




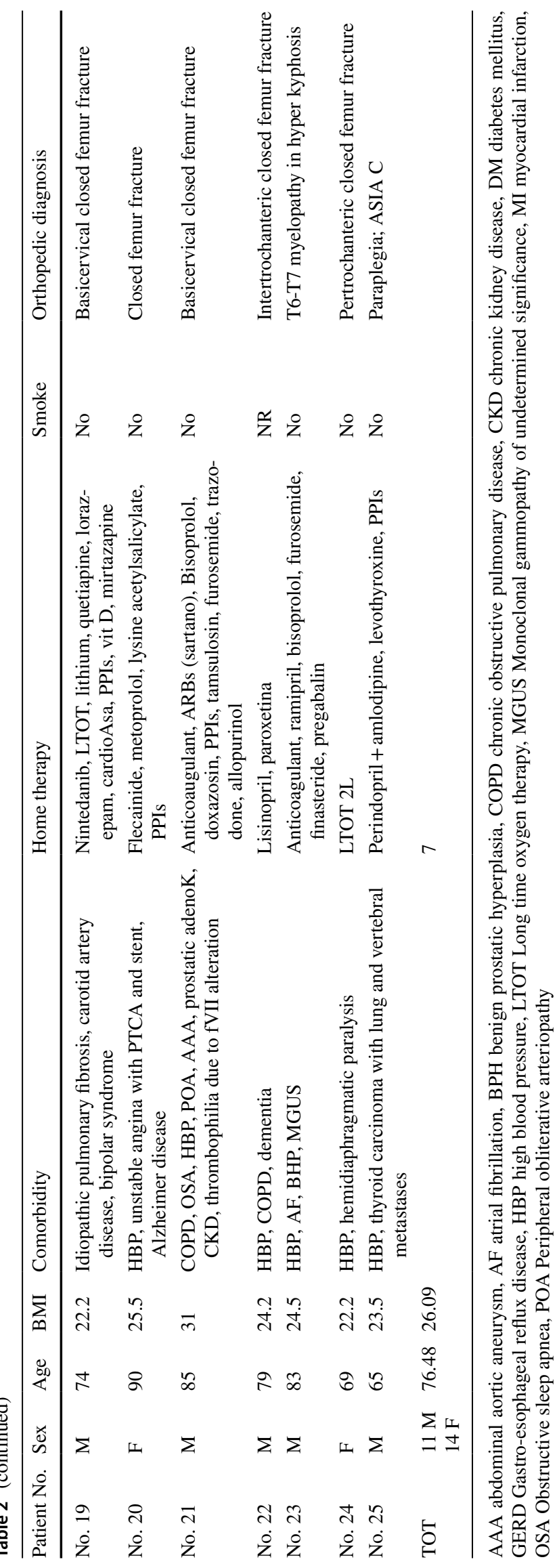




\section{Clinical presentation}

Out of the 25 cases, seven were defined as suspected before hospitalization. Of these, six had respiratory symptoms. Three reported a direct contact with Covid-19 positive patients (Fig. 2).

Eighteen patients had no typical symptoms at hospital admission. Sixteen out of 18 developed symptoms during the hospital stay, while two remained asymptomatic. One patient become symptomatic after discharge, requiring admission to a COVID hospital. Another patient (\#12) was diagnosed for COVID disease while performing a thoracic CT scan for the study of an underlying disease (Osteosarcoma) (Fig. 2).

The most frequently reported symptoms included: fever, cough, dyspnea, and diarrhea, recurrent in 18, 13, 12, and five cases, respectively. Nineteen severe episodes of desaturation requiring oxygen therapy were recorded. All signs and symptoms are reported in Table 3.

\section{Role of swab tests and CT scan}

Seventeen of the 18 non-suspected patients were placed in the standard orthopedic ward. One patient before hospitalization had CT signs of pulmonary fibrosis despite a negative swab test but was hospitalized in the COVID-19 ward as a precaution. Figure 3 describes the access management to the COVID-19 ward. The timing of each patient's relocation based on tests results is shown in Table 4.

From a diagnostic perspective, only two of 25 cases were positive to the NP and OP swabs without having a suspected CT picture; seven of 25 patients resulted positive to NP and OR swab tests while showing a typical CT scan (on the 50\% of the cases, the positive outcome of the swab test followed the suspected CT outcomes), 14 of 25 patients had only a suspected CT scan and were negative to the swabs tests, a single case had negative results in both tests, while only one had not in hospital screening and he was identified after discharge. Three of 25 patients were diagnosed or suspected before surgical procedure.

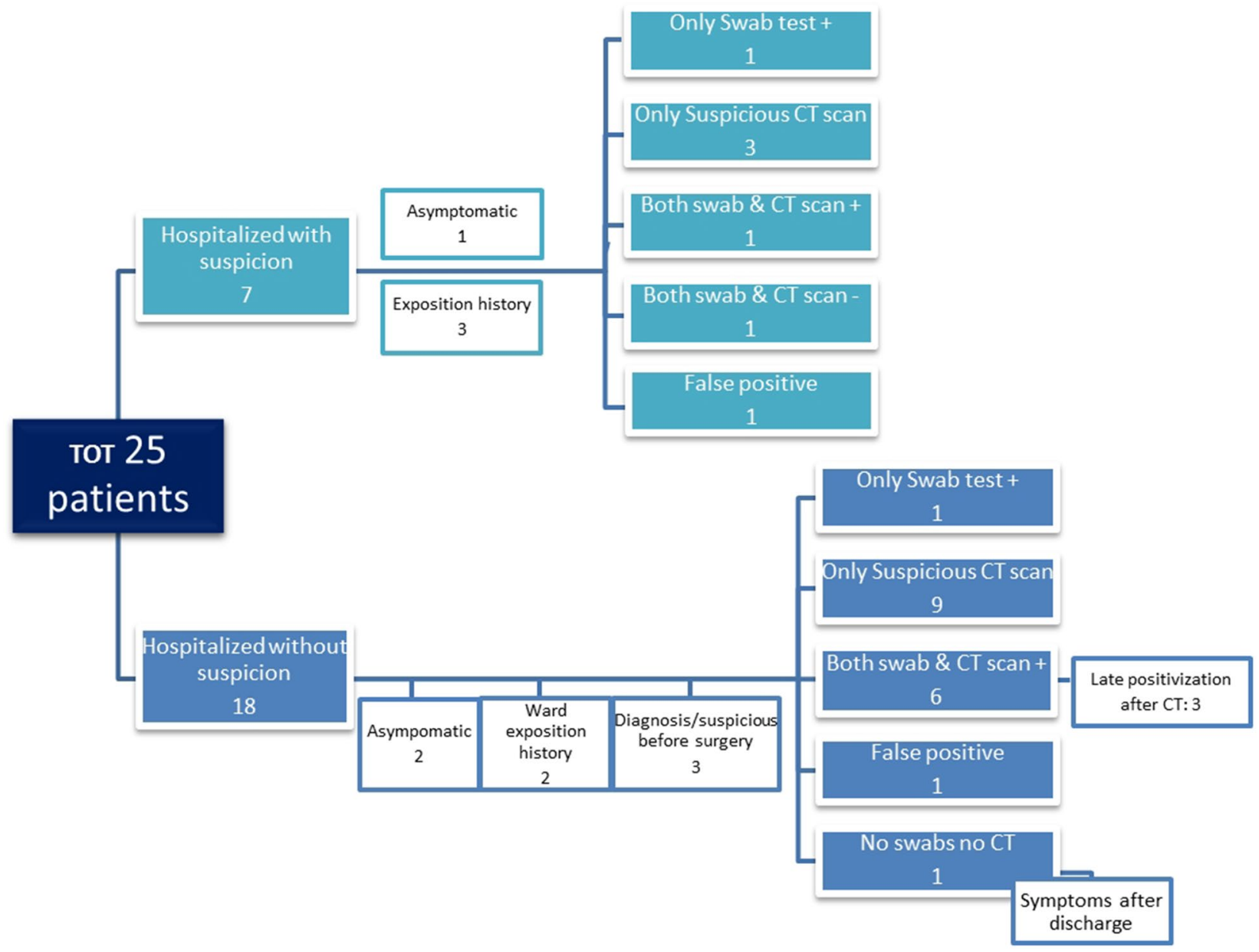

Fig. 2 Comprehensive flowchart concerning patient's management 
MUSCULOSKELETAL SURGERY (2022) 106:407-426

413

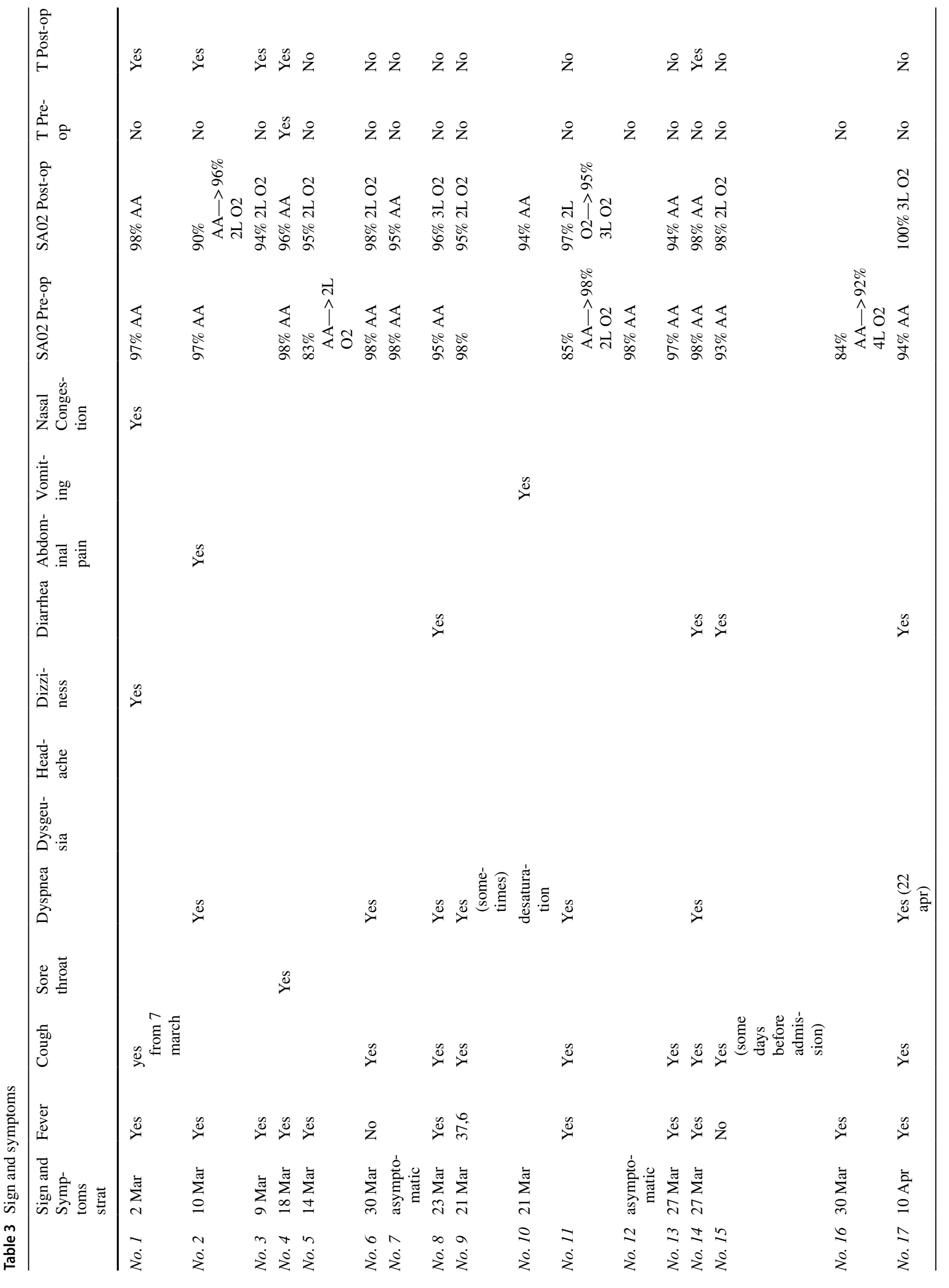

Springer 
414

MUSCULOSKELETAL SURGERY (2022) 106:407-426

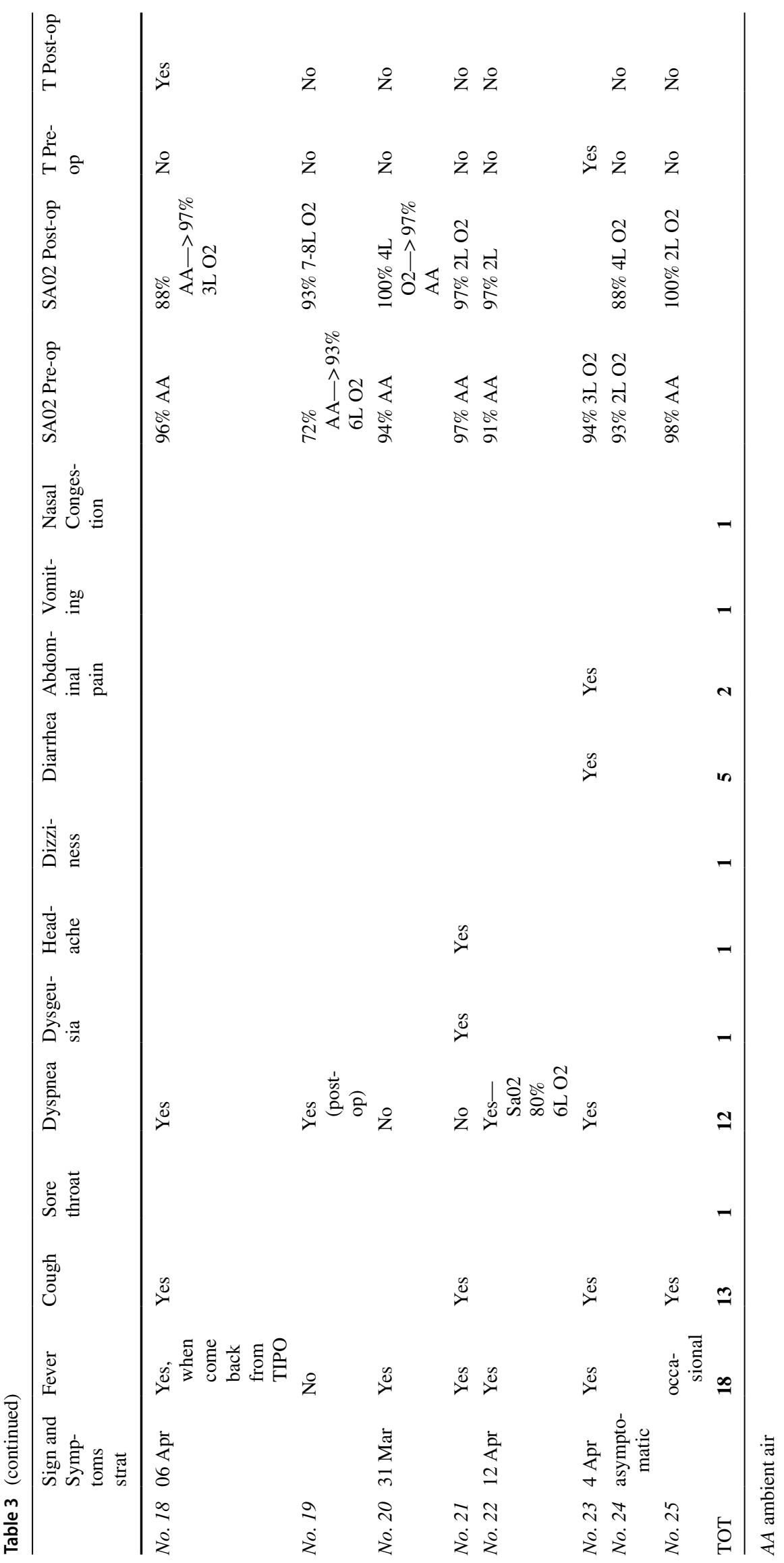

Springer 


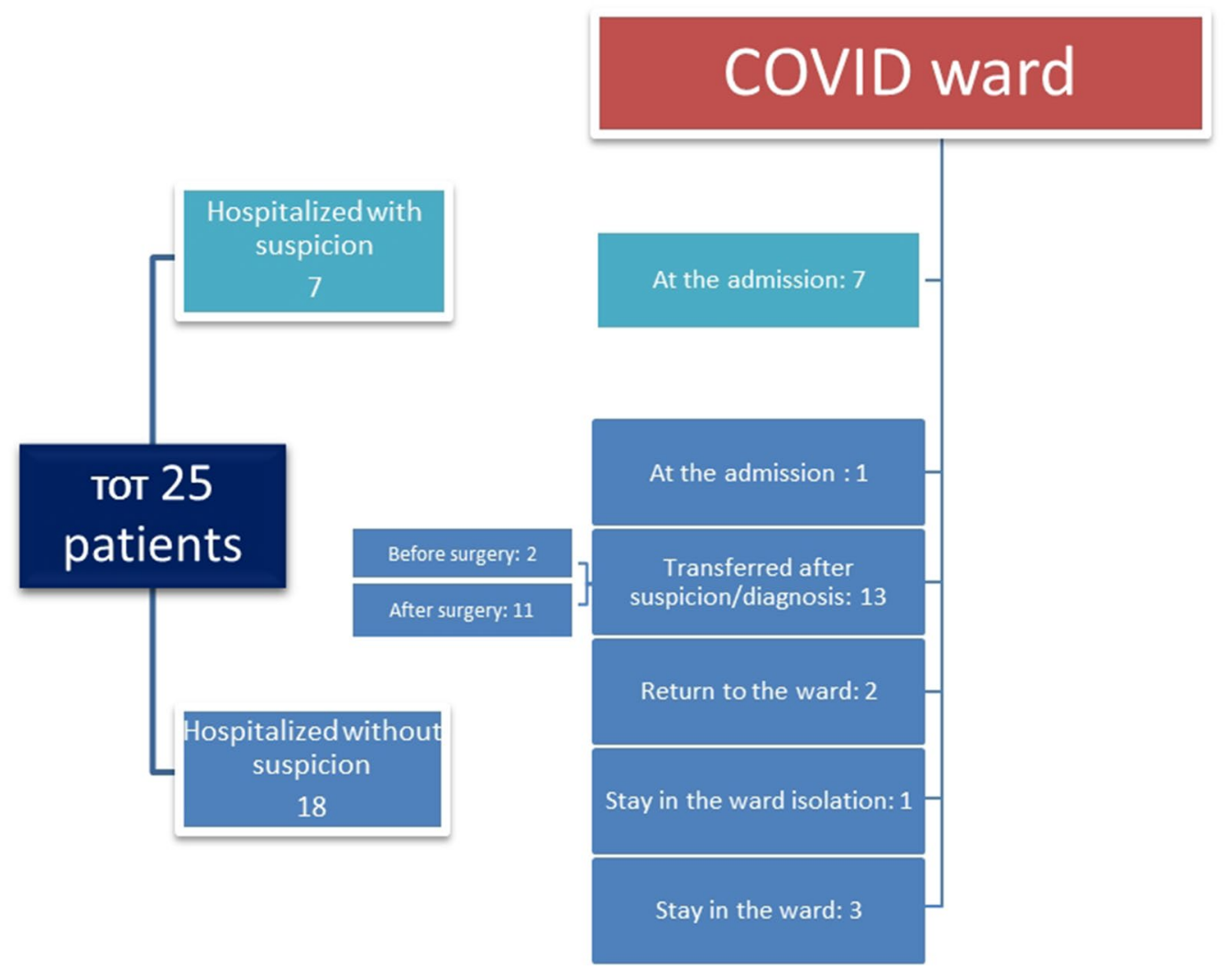

Fig. 3 Accesses to COVID ward

Out of the 25 cases, two false positives were found; in particular, out of the 14 cases with suspected CT findings, in two cases the diagnosis changed over time: one diagnosis lead to a cardiopulmonary congestion, another to a chronic obstructive pulmonary disease (COPD) exacerbation.

In total there were nine positive swabs tests and 14 cases with suspected CT scan findings with a negative swab test (Table 5).

In detail, among the nine confirmed COVID-19 positive patients, female gender prevails in a ratio of 6:3. The average age was 71.3 years (range 34-96), and a tendency toward overweight was found (average BMI 27.6; range 20.8-35.2). The 14 patients with a suspected CT scan had similar gender distribution (7:7) with an average age of 78.3 years (range 34-96) and average normal weight (24.8 BMI; range 21.6-26.9). The time to formulate the COVID-19 infection diagnosis by CT scan was about four days faster than the one with utilized of swabs. The time to onset of symptoms from admission or surgical procedure was similar between the first and the second group. There was no clear prevalence of comorbidity or pathology or surgical procedure type between the two groups, but surgical time was longer in confirmed positive COVID-19 patients.

\section{Main laboratory findings}

The laboratory results are shown in Table 6. Reported data refer to the day of the NP and OP swab test positive outcome or, for those patients with negative swabs tests, to the day of the CT examination with suspected results.

Several laboratory changes have been reported during hospitalization since the onset of symptoms (Table 7): anemia $(n=24)$, persisting over 5 days [10] in 15 patients (threshold refers to the anemia low peak usually occurring after three-four postoperative days), leukocytosis $(n=5)$, with an increase in neutrophils in all patients, and of monocytes in three; 13 patients had lymphopenia, four had numerical alteration of platelets (one thrombocytosis and three thrombocytopenia), two showed coagulation abnormalities, three showed an increase in the levels of alkaline phosphatase, three patients presented an increase in liver enzymes, while all had an increase in C-reactive protein.

The laboratory changes reported in the confirmed COVID-19 cases occurred in a period ranging from the first postoperative day (POD) to the eleventh, with an average of 3.3 days, while in the suspected COVID-19 group blood tests alterations occurred earlier, already from the first POD. 
Table 4 Timing and modality of diagnosis and evidence of COVID-19

\begin{tabular}{|c|c|c|c|c|c|c|c|c|c|c|c|}
\hline & $\begin{array}{l}\text { EXPOSURE } \\
\text { HISTORY }\end{array}$ & $\begin{array}{l}\text { SUSPECTED } \\
\text { AT THE } \\
\text { ADMISSION }\end{array}$ & $\begin{array}{l}\text { PRE-OP } \\
\text { DIAGNOSIS }\end{array}$ & $\begin{array}{l}\text { DAY SINCE } \\
\text { ADMISSIO } \\
\mathrm{N} \text { TO } \\
\text { SYMPTOMS }\end{array}$ & $\begin{array}{l}\text { DAY SINCE } \\
\text { SURGERY } \\
\text { TO } \\
\text { SYMPTOMS }\end{array}$ & $\begin{array}{l}\text { DAY SINCE } \\
\text { ADMISSION } \\
\text { TO } \\
\text { DIAGNOSIS/S } \\
\text { USPECT }\end{array}$ & $\begin{array}{c}\text { DAY SINCE SURGERY } \\
\text { TO } \\
\text { DIAGNOSIS/SUSPECT }\end{array}$ & $\begin{array}{l}\text { WARD } \\
\text { BEFORE } \\
\text { SURGERY }\end{array}$ & $\begin{array}{l}\text { WARD } \\
\text { AFTER } \\
\text { SURGERY }\end{array}$ & $\begin{array}{c}\text { SARS-COV } \\
\text { QUANTITATIVE } \\
\text { RT-PCR }\end{array}$ & $\begin{array}{l}\text { TYPICAL } \\
\text { SIGNS OF } \\
\text { VIRAL } \\
\text { INFECTION } \\
\text { ON CT }\end{array}$ \\
\hline \#1 & NR & no & no & 4 & 5 & 13 & 12 & $\begin{array}{l}\text { orthoped } \\
\text { ic ward }\end{array}$ & $\begin{array}{l}\text { orthopedic } \\
\text { ward } \\
\text { (isolation) }\end{array}$ & positive & bilateral \\
\hline$\# 2$ & NR & no & no & 6 & 1 & 11 & 6 & $\begin{array}{l}\text { orthoped } \\
\text { ic ward }\end{array}$ & $\begin{array}{l}\text { orthopedic } \\
\text { ward -> } \\
\text { COVID } \\
\text { section }\end{array}$ & positive & bilateral \\
\hline \#3 & NR & no & no & 0 & -1 & 8 & 7 & $\begin{array}{l}\text { orthoped } \\
\text { ic ward }\end{array}$ & $\begin{array}{l}\text { orthopedic } \\
\text { ward } \\
\text { (isolation) }\end{array}$ & positive & bilateral \\
\hline$\# 4$ & NR & no & $\begin{array}{l}\text { only for } \\
\text { second } \\
\text { operation }\end{array}$ & 8 & 7 & 13 & 12 & $\begin{array}{l}\text { orthoped } \\
\text { ic ward }\end{array}$ & $\begin{array}{c}\text { COVID } \\
\text { section -> } \\
\text { orthopedic } \\
\text { ward }\end{array}$ & Positive & negative \\
\hline$\# \mathbf{5}$ & $\begin{array}{l}\text { exposure to } \\
\text { suspected } \\
\text { case }\end{array}$ & yes & yes & 0 & -3 & 3 & $\begin{array}{l}\text { diagnosis pre-op: } 1 \\
\text { day before the first } \\
\text { operation }\end{array}$ & $\begin{array}{l}\text { COVID } \\
\text { section }\end{array}$ & $\begin{array}{l}\text { COVID } \\
\text { section }\end{array}$ & positive & negative \\
\hline$\# 6$ & NR & no & no & 15 & 11 & $\begin{array}{l}4 \text { (only CT } \\
\text { suspected) }\end{array}$ & $\begin{array}{l}\text { CT suspect same days } \\
\text { of surgery }\end{array}$ & $\begin{array}{l}\text { orthopedi } \\
\mathrm{c} \text { ward }\end{array}$ & $\begin{array}{l}\text { COVID } \\
\text { section }\end{array}$ & negative & bilateral \\
\hline$\# 7$ & NR & no & no & I & 1 & no & no & $\begin{array}{l}\text { orthopedi } \\
\text { c ward }\end{array}$ & $\begin{array}{l}\text { orthopedic } \\
\text { ward }\end{array}$ & not done & not done \\
\hline$\# 8$ & NR & no & no & 6 & 5 & $\begin{array}{l}6 \text { (only CT } \\
\text { suspected) }\end{array}$ & 5 (CT suspect) & $\begin{array}{l}\text { orthopedi } \\
\text { c ward }\end{array}$ & $\begin{array}{l}\text { COVID } \\
\text { section }\end{array}$ & negative & bilateral \\
\hline$\# 9$ & NR & no & no & 3 & 2 & $\begin{array}{l}3 \text { (symptoms } \\
\text { suspeted) }\end{array}$ & 2 (CT suspect) & $\begin{array}{l}\text { orthopedi } \\
\text { c ward }\end{array}$ & $\begin{array}{l}\text { orthopedic } \\
\text { ward }\end{array}$ & negative & $\begin{array}{l}\text { bilateral -> } \\
\text { rectified in } \\
\text { BPCO flare }\end{array}$ \\
\hline$\# 10$ & $\begin{array}{l}\text { exposure to } \\
\text { relevant } \\
\text { environmen } \\
t\end{array}$ & yes & suspect & 0 & -3 & $\begin{array}{l}1 \text { (only CT } \\
\text { suspected) }\end{array}$ & $\begin{array}{l}\text { Ct suspect pre-op: } 2 \\
\text { day before surgery }\end{array}$ & $\begin{array}{l}\text { COVID } \\
\text { section }\end{array}$ & $\begin{array}{l}\text { COVID } \\
\text { section }\end{array}$ & negative & bilateral \\
\hline \# 11 & NR & yes & yes & 0 & -2 & 2 & $\begin{array}{l}\text { Diagnosis pre-op:1 } \\
\text { day before surgery }\end{array}$ & $\begin{array}{l}\text { COVID } \\
\text { section }\end{array}$ & $\begin{array}{l}\text { COVID } \\
\text { section }\end{array}$ & positive & bilateral \\
\hline \# 12 & NR & yes & 1 & 1 & 1 & $\begin{array}{l}1 \text { (only CT } \\
\text { suspected) }\end{array}$ & 1 & $\begin{array}{l}\text { COVID } \\
\text { section }\end{array}$ & 1 & negative & Bilateral \\
\hline \# 13 & $\begin{array}{l}\text { contact with } \\
\text { healthcare } \\
\text { staff swab } \\
\text { positive }\end{array}$ & no & no & 2 & 0 & $\begin{array}{l}5 \text { (only CT } \\
\text { suspected) }\end{array}$ & 3 (CT suspect) & $\begin{array}{l}\text { orthopedi } \\
\text { c ward }\end{array}$ & $\begin{array}{l}\text { COVID } \\
\text { section -> } \\
\text { orthopedic } \\
\text { ward }\end{array}$ & negative & bilateral \\
\hline \# 14 & NR & no & no & 1 & 1 & $\begin{array}{l}4 \text { (symptoms } \\
\text { suspected) }\end{array}$ & 4 (CT suspect) & $\begin{array}{l}\text { orthopedi } \\
\text { c ward }\end{array}$ & $\begin{array}{l}\text { COVID } \\
\text { section }\end{array}$ & negative & monolateral \\
\hline \# 15 & NR & no & suspect & 0 & -2 & $\begin{array}{l}1 \text { (only CT } \\
\text { suspected) }\end{array}$ & $\begin{array}{l}\text { Diagnosis pre-op: } 2 \\
\text { day before surgery }\end{array}$ & $\begin{array}{l}\text { orthopedi } \\
\text { c ward }\end{array}$ & $\begin{array}{c}\text { orthopedic } \\
\text { ward -> } \\
\text { COVID } \\
\text { section }\end{array}$ & negative & monolateral \\
\hline$\# 16$ & NR & no & suspect & 2 & -7 & $\begin{array}{l}2 \text { (only CT } \\
\text { suspected) }\end{array}$ & 7 (CT suspect) & $\begin{array}{l}\text { orthopedi } \\
\text { c ward -> } \\
\text { COVID } \\
\text { section }\end{array}$ & $\begin{array}{l}\text { COVID } \\
\text { section }\end{array}$ & Negative & monolateral \\
\hline \# 17 & $\begin{array}{l}\text { exposure in } \\
\text { ICU with pt } \\
\text { covid }+\end{array}$ & no & no & 11 & 7 & 12 & 8 & $\begin{array}{l}\text { orthoped } \\
\text { ic ward }\end{array}$ & $\begin{array}{l}\text { orthopedic } \\
\text { ward } \\
\text { (isolation) - } \\
>\text { COVID } \\
\text { section }\end{array}$ & positive & bilateral \\
\hline
\end{tabular}


Table 4 (continued)

\begin{tabular}{|c|c|c|c|c|c|c|c|c|c|c|c|}
\hline \# 18 & NR & no & no & 6 & 4 & 8 & 5 & $\begin{array}{l}\text { orthoped } \\
\text { ic ward }\end{array}$ & $\begin{array}{l}\text { Covid } \\
\text { section }\end{array}$ & positive & monolateral \\
\hline$\# 19$ & $\mathrm{NR}$ & yes & suspect & 1 & 1 & $\begin{array}{c}\text { same day } \\
\text { (symptoms \& } \\
\text { CT } \\
\text { suspected) }\end{array}$ & $\begin{array}{l}\text { same day (CT } \\
\text { suspect) }\end{array}$ & $\begin{array}{l}\text { COVID } \\
\text { section }\end{array}$ & $\begin{array}{l}\text { CoVID } \\
\text { section }\end{array}$ & negative & bilateral \\
\hline$\# 20$ & NR & yes & suspect & 0 & -1 & $\begin{array}{c}\text { same day } \\
\text { (symptoms \& } \\
\text { CT } \\
\text { suspected) }\end{array}$ & $\begin{array}{l}\text { CT suspect pre-op: } 1 \\
\text { day before surgery }\end{array}$ & $\begin{array}{l}\text { COVID } \\
\text { section }\end{array}$ & $\begin{array}{l}\text { CoVID } \\
\text { section }\end{array}$ & negative & $\begin{array}{c}\text { monolateral } \\
\rightarrow>\text { rectified } \\
\text { in } \\
\text { in } \\
\text { cardiopulmo } \\
\text { nary } \\
\text { congestion }\end{array}$ \\
\hline$\# 21$ & $\begin{array}{c}\text { exposure to } \\
\text { relevant } \\
\text { environmen } \\
t\end{array}$ & yes & suspect & 0 & -2 & $\begin{array}{l}\text { same day } \\
\text { (only } \\
\text { symptoms } \\
\text { suspected) }\end{array}$ & $\begin{array}{l}\text { only symptoms } \\
\text { suspected pre-op: } 2 \\
\text { days before surgery }\end{array}$ & $\begin{array}{l}\text { COVID } \\
\text { section }\end{array}$ & $\begin{array}{l}\text { COVID } \\
\text { section }\end{array}$ & Negative & Negative \\
\hline \#22 & NR & $\begin{array}{l}\text { no (negative } \\
\text { swab do at } \\
\text { aid of } \\
\text { origin) }\end{array}$ & suspect & 10 & 9 & $\begin{array}{l}10 \text { (only CT } \\
\text { suspected) }\end{array}$ & 9 (CT suspect) & $\begin{array}{l}\text { COVID } \\
\text { section }\end{array}$ & $\begin{array}{c}\text { COVID } \\
\text { section - } \\
\text { transferred } \\
\text { in } \\
\text { orthopedic } \\
\text { ward cause } \\
\text { no Covid } \\
\text { suspect- } \\
\text { return to } \\
\text { coVID } \\
\text { section } \\
\text { cause } \\
\text { clinical } \\
\text { worsening }\end{array}$ & negative & bilateral \\
\hline$\# 23$ & NR & no & 1 & 1 & 1 & $\begin{array}{c}1 \text { (only } \\
\text { clinical \& CT } \\
\text { suspected) }\end{array}$ & 1 & $\begin{array}{l}\text { orthopedi } \\
\text { Cward-s } \\
\text { COVVD } \\
\text { section }\end{array}$ & 1 & negative & bilateral \\
\hline \# 24 & NR & no & no & 1 & 1 & $\begin{array}{l}1 \text { (only CT } \\
\text { suspected) }\end{array}$ & 1 (CT suspected) & $\begin{array}{l}\text { orthopedi } \\
\mathrm{c} \text { ward }\end{array}$ & $\begin{array}{l}\text { COVID } \\
\text { section }\end{array}$ & negative & bilateral \\
\hline$\# 25$ & NR & no & no & 0 & 0 & 1 & 1 & $\begin{array}{l}\text { orthoped } \\
\text { ic ward }\end{array}$ & $\begin{array}{l}\text { Covid } \\
\text { section }\end{array}$ & positive & monolateral \\
\hline тот & 5 & 7 & $\begin{array}{l}7 \text { suspect- } \\
2 \text { diagnosis }\end{array}$ & & & & & & & 9 & $\begin{array}{c}19+2 \text { false } \\
\text { positive }\end{array}$ \\
\hline AVERAGE & & & & 3.4 & 1.5 & 4.6 & 3.3 & & & & \\
\hline
\end{tabular}

Orange: positive swab and CT scan; Red: only positive swab; White: only CT scan compatible; Cyan: false positive; Grey: no tests; Purple: both negative tests

Table 5 Comparison among confirmed and suspected COVID-19 cases

\begin{tabular}{lll}
\hline & Confirmed cases: 9 & Suspected cases: 14 \\
\hline Gender & $3 \mathrm{M}-6 \mathrm{~F}$ & $7 \mathrm{M}-7 \mathrm{~F}$ \\
Age & 71.3 & 78.3 \\
BMI & 27.6 & 24.8 \\
Day since admission to symptoms & 3.88 & 3.45 \\
Day since surgery to symptoms & 2 & 1.3 \\
Day since admission to diagnosis/suspect & 7.88 & 2.78 \\
Day since surgery to diagnosis/suspect & 5.44 & 2.16 \\
Surgery time (h) & $02: 45$ & $01: 10$ \\
\hline
\end{tabular}

Average values

\section{Patients' management and ICU access}

Patients were operated on orthopedic pathology as reported in Table 8. Fifteen patients received antibiotic therapy in relation to postoperative protocols or for infective complication other than COVID-19. Intensive care recovery was required for three patients during the first postoperative period for monitoring clinical conditions. All the patients underwent anti-thromboembolic 


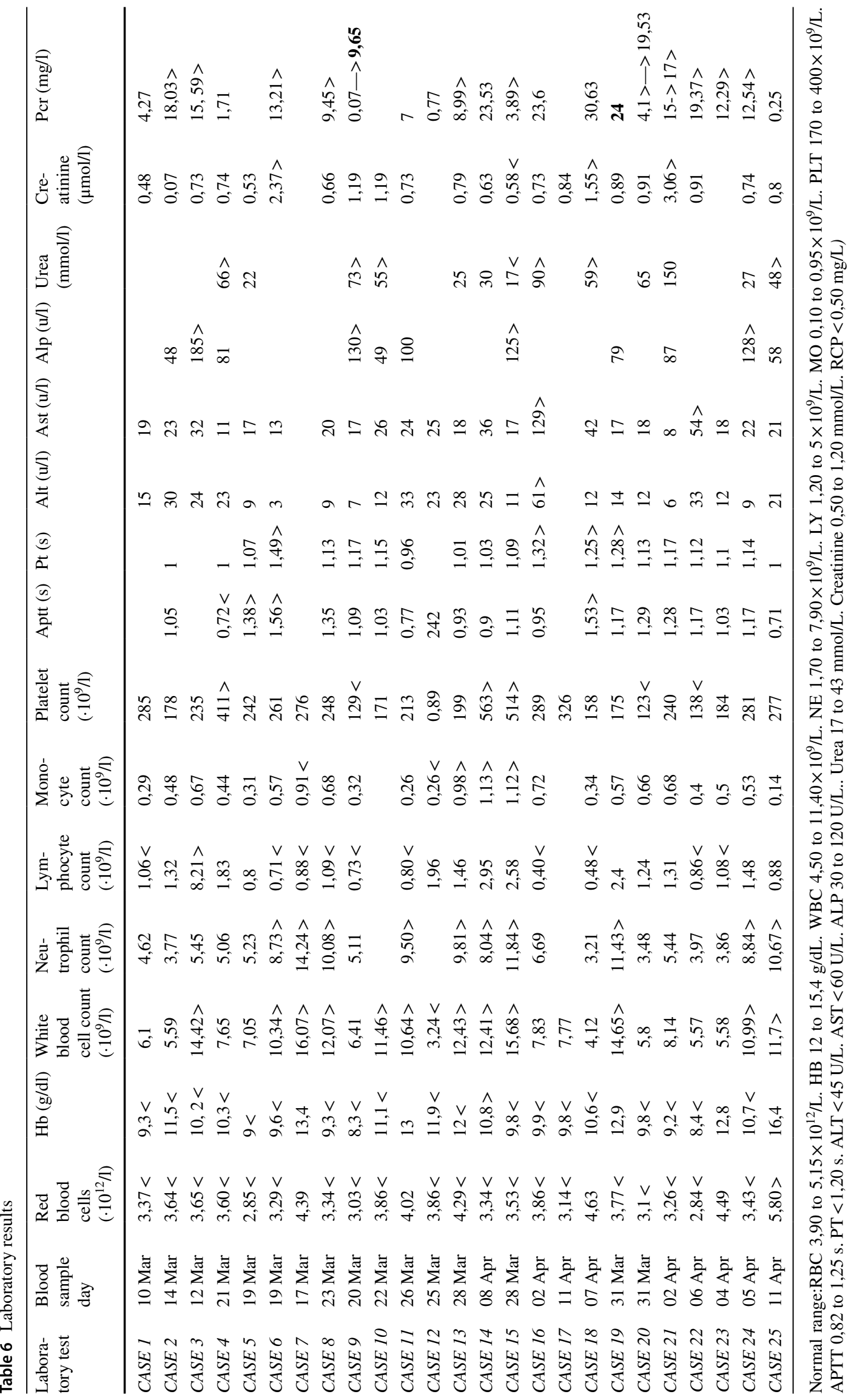


Table 7 Orange: positive swab and CT scan; Red: only positive swab; White: only CT scan compatible; Cyan: false positive; Gray: no tests; Purple: both negative tests

\begin{tabular}{|c|c|c|c|c|c|c|c|c|c|}
\hline $\begin{array}{l}\text { LABORATORY } \\
\text { TEST }\end{array}$ & $\begin{array}{c}\text { ANEMIA } \\
\text { POST-OP } \\
\text { OVER } 5 \\
\text { DAYS }\end{array}$ & LEUKOCYTOSIS & LYMPHOCYTOSIS & LINFOPENIA & THROMBOCYTOSIS & THROMBOCYTOPENIA & $\begin{array}{l}\text { COAGULATION } \\
\text { DISORDERS }\end{array}$ & $\begin{array}{c}\text { LIVER } \\
\text { ENZYME } \\
\text { CHANGES }\end{array}$ & $\begin{array}{l}\text { INCREASE } \\
\text { IN ALP }\end{array}$ \\
\hline$\# 1$ & yes & & & from 11 POD & & & & & \\
\hline$\# 2$ & yes & & & 2 POD & & & & & \\
\hline$\# 3$ & yes & & from 6 POD & & & & & $\begin{array}{l}\text { from } 6 \\
\text { POD }\end{array}$ & $\begin{array}{c}\text { From } 4 \\
\text { POD }\end{array}$ \\
\hline$\# 4$ & yes & & & & from 1 POD & & & & \\
\hline$\# 5$ & & & & from 1 POD & & & & & \\
\hline$\# 6$ & & from 1 POD & & from 1 POD & & & from $1 \mathrm{POD}$ & & \\
\hline \multicolumn{10}{|l|}{$\# 7$} \\
\hline$\# 8$ & yes & from 1 POD & & from 1 POD & & & & & \\
\hline$\# 9$ & & & & $1 \mathrm{POD}$ & & & & & From 1 POD \\
\hline \multicolumn{10}{|l|}{$\# 10$} \\
\hline$\# 11$ & yes & & & from 1 POD & & & & $\begin{array}{l}\text { from } 4 \\
\text { POD }\end{array}$ & \\
\hline \multicolumn{10}{|l|}{ \# 12} \\
\hline \multicolumn{10}{|l|}{ \# 13} \\
\hline \# 14 & yes & from 1 POD & from 1 POD & & & & & & \\
\hline \multicolumn{10}{|l|}{ \# 15} \\
\hline \# 16 & & & & $\begin{array}{l}\text { pre-op with } \\
\text { symptoms }\end{array}$ & & & & & \\
\hline$\# 17$ & yes & & & from 1 POD & & & & & \\
\hline \multicolumn{10}{|l|}{$\# 19$} \\
\hline$\# 20$ & yes & & & & & from 1 POD & & & \\
\hline \multicolumn{10}{|l|}{$\# 21$} \\
\hline \# 22 & yes & from $1 P O D$ & & from $1 P O D$ & & & & & \\
\hline \#23 & $\begin{array}{l}\text { yes (no } \\
\text { surgery) }\end{array}$ & & & $\begin{array}{l}\text { yes (no } \\
\text { surgery) }\end{array}$ & & & & & \\
\hline$\# 24$ & & & & & & & & & From 1 POD \\
\hline$\# 25$ & & from 1 POD & & $\begin{array}{l}\text { yes (same } \\
\text { day of } \\
\text { surgery) } \\
\end{array}$ & & 2-3 POD & & $\begin{array}{c}\text { from } 9 \\
\text { POD }\end{array}$ & \\
\hline тот & 11 & 5 & 2 & 13 & 1 & 3 & 2 & 3 & 3 \\
\hline
\end{tabular}

prophylaxis according to the institutional protocols. Twelve patients received blood transfusions.

Nineteen patients were treated by oxygen supplement, seven of these required continuous positive airway pressure (CPAP), and no patients required invasive mechanical ventilation outside the postoperative intensive care. Glucocorticoids were administered in two patients. Three patients were administered antivirals, eight with antibiotic therapy, and nine with hydroxychloroquine (Plaquenil®); those who were administered antiviral therapy were at the same time treated by antibiotic, hydroxychloroquine, or both. The pharmacology therapy utilized are specified in Table 9.

\section{Clinical course and outcomes}

Final outcomes were: five dead patients, seven transferred to COVID-19 hospital, nine discharged at home after 14 days of isolation in the dedicated ward, three discharged at home or in nursing home in isolation, one discharged, and then readmitted to the COVID-19 reference hospital. Complications occurred in 16 patients. One patient developed a postoperative infection (Table 10).

With regard to the deceased patients, the following common features were identified: All patients were over 74 years old with multiple comorbidities, all were tested negative for NP and OP swabs, four out of five had suspected CT 
Table 8 Patients management and ICU access

\begin{tabular}{|c|c|c|c|c|c|c|c|c|}
\hline & $\begin{array}{l}\text { Orthopedics } \\
\text { surgery }\end{array}$ & $\begin{array}{l}\text { Date of } \\
\text { operation }\end{array}$ & $\begin{array}{l}\text { Surgery time } \\
\text { (h) }\end{array}$ & Antibiotics post-op & $\begin{array}{l}\text { Need for } \\
\text { pre-op } \\
\text { ICU }\end{array}$ & $\begin{array}{l}\text { Need for } \\
\text { post-op ICU }\end{array}$ & LMWH use & Transfusion \\
\hline No. 1 & $\begin{array}{l}\text { Total hip } \\
\text { arthroplasty }\end{array}$ & $28 / 02 / 2020$ & $2: 20$ & $\begin{array}{l}\text { Cefamezin (for cooling symp- } \\
\text { toms) }\end{array}$ & & & Yes & 1 \\
\hline No. 2 & $\begin{array}{l}\text { Herniec- } \\
\text { tomy and } \\
\text { posterior } \\
\text { vertebral } \\
\text { arthrodesis } \\
\text { L3-L4 }\end{array}$ & $09 / 03 / 2020$ & $2: 53$ & & No & No & Yes & No \\
\hline No. 3 & $\begin{array}{l}\text { Hip hemiar- } \\
\text { throplasty }\end{array}$ & $10 / 03 / 2020$ & $1: 20$ & Tazocin (for urinary infection) & No & No & Yes & No \\
\hline No. 4 & $\begin{array}{l}\text { Lleg amputa- } \\
\text { tion and } \\
\text { revisions }\end{array}$ & $\begin{array}{l}11 \& \\
27 / 03 / 2020 \\
\& 14-04- \\
2020\end{array}$ & $\begin{array}{l}01: 30 \& \\
00: 20 \& \\
01: 00\end{array}$ & Tazocin & No & No & Yes & No \\
\hline No. 5 & $\begin{array}{l}\text { Cotile reim- } \\
\text { plantation }\end{array}$ & $\begin{array}{l}17 \& \\
20 / 03 / 2020\end{array}$ & $01: 55 \& 1: 20$ & No & No & No & Yes & 6 bag post-op \\
\hline No. 6 & $\begin{array}{l}\text { Hip hemiar- } \\
\text { throplasty }\end{array}$ & $19 / 03 / 2020$ & $1: 40$ & $\begin{array}{l}\text { Tazocin } \longrightarrow \text { Klacid } \longrightarrow \text { Mero- } \\
\text { penem }\end{array}$ & No & No & Yes & 4 \\
\hline No. 7 & $\begin{array}{l}\text { Osteosyn- } \\
\text { thesis } \\
\text { intramedu- } \\
\text { lary rod }\end{array}$ & $17 / 03 / 2020$ & $0: 50$ & No & No & No & Yes & No \\
\hline No. 8 & $\begin{array}{l}\text { Osteosyn- } \\
\text { thesis } \\
\text { intramedu- } \\
\text { lary rod }\end{array}$ & $18 / 03 / 2020$ & 1:07 & Tazocin + claritromicina & No & No & Yes & 2 \\
\hline No. 9 & $\begin{array}{l}\text { Osteosyn- } \\
\text { thesis } \\
\text { intramedu- } \\
\text { lary rod }\end{array}$ & $19 / 03 / 2020$ & $1: 10$ & Tazocin & No & No & Yes & 3 \\
\hline No. 10 & $\begin{array}{l}\text { Distal radius } \\
\text { external } \\
\text { fixator }\end{array}$ & $24 / 03 / 2020$ & $1: 44$ & clindamicina $\longrightarrow$ levoxacin & No & No & Yes & No \\
\hline No. 11 & $\begin{array}{l}\text { Total hip } \\
\text { arthroplasty }\end{array}$ & $26 / 03 / 2020$ & 1:07 & No & No & No & Yes & No \\
\hline No. 12 & $\begin{array}{l}\text { CT osteosar- } \\
\text { choma }\end{array}$ & $24 / 03 / 2020$ & & & No & No & Yes & No \\
\hline No. 13 & $\begin{array}{l}\text { Surgical } \\
\text { wound revi- } \\
\text { sion }\end{array}$ & $27 / 03 / 2020$ & $0: 23$ & Tazocin + clindamicina & No & No & Yes & No \\
\hline No. 14 & $\begin{array}{l}\text { Hip hemiar- } \\
\text { throplasty }\end{array}$ & $26 / 03 / 2020$ & 1:00 & Tazocin + claritromicina & No & No & Yes & No \\
\hline No. 15 & $\begin{array}{l}\text { Osteosyn- } \\
\text { thesis } \\
\text { intramedu- } \\
\text { lary rod }\end{array}$ & $30 / 03 / 2020$ & $0: 40$ & Tazocin + Claritromicina & No & No & Yes & 1 \\
\hline No. 16 & $\begin{array}{l}\text { Osteosyn- } \\
\text { thesis } \\
\text { intramedu- } \\
\text { lary rod }\end{array}$ & $06 / 04 / 2020$ & $1: 34$ & & No & No & & \\
\hline No. 17 & $\begin{array}{l}\text { Posterior } \\
\text { artrodesis } 5 \\
\text { levels }\end{array}$ & 03/04/2020 & $4: 40$ & $\begin{array}{l}\text { Tazocin for suspected } \\
\text { HAP } \longrightarrow \text { Azitromicina }\end{array}$ & No & $\begin{array}{l}\text { Yes, orthope- } \\
\text { dic reason, } \\
3 \text { days }\end{array}$ & Yes & 2 \\
\hline
\end{tabular}


Table 8 (continued)

\begin{tabular}{|c|c|c|c|c|c|c|c|c|}
\hline & $\begin{array}{l}\text { Orthopedics } \\
\text { surgery }\end{array}$ & $\begin{array}{l}\text { Date of } \\
\text { operation }\end{array}$ & $\begin{array}{l}\text { Surgery time } \\
\text { (h) }\end{array}$ & Antibiotics post-op & $\begin{array}{l}\text { Need for } \\
\text { pre-op } \\
\text { ICU }\end{array}$ & $\begin{array}{l}\text { Need for } \\
\text { post-op ICU }\end{array}$ & LMWH use & Transfusion \\
\hline No. 18 & $\begin{array}{l}\text { Embolization } \\
\text { \& arthrode- } \\
\text { sis } 3 \text { levels } \\
\text { lumbar } \\
\text { spine }\end{array}$ & $\begin{array}{l}01- \\
02 / 04 / 2020\end{array}$ & $7: 27$ & Tazocin & No & $\begin{array}{l}\text { Yes, orthope- } \\
\text { dic reason, } \\
\text { for } 4 \text { day }\end{array}$ & Yes & No \\
\hline No. 19 & $\begin{array}{l}\text { Total hip } \\
\text { arthroplasty }\end{array}$ & $31 / 03 / 2020$ & $0: 30$ & $\begin{array}{l}\text { Tazocin + azitromicina + Lin- } \\
\text { ezolid }\end{array}$ & No & No & Yes & 2 bag post-op \\
\hline No. 20 & $\begin{array}{l}\text { Hip hemiar- } \\
\text { throplasty }\end{array}$ & $01 / 04 / 2020$ & 1:07 & $\begin{array}{l}\text { azitromicina }+ \text { ceftriaxone }- \\
\text { ceftriaxone }>\text { Meropenem }\end{array}$ & No & No & Yes & 2 \\
\hline No. 21 & $\begin{array}{l}\text { Hip hemiar- } \\
\text { throplasty }\end{array}$ & 03/04/2020 & $2: 20$ & No & No & No & Yes & 2 \\
\hline No. 22 & $\begin{array}{l}\text { Plate and } \\
\text { screws } \\
\text { osteosyn- } \\
\text { thesis }\end{array}$ & 03/04/2020 & $1: 12$ & No & No & No & Yes & 2 \\
\hline No. 23 & Not done & & & & & & & \\
\hline No. 24 & $\begin{array}{l}\text { Osteosyn- } \\
\text { thesis } \\
\text { intramedu- } \\
\text { lary rod }\end{array}$ & $04 / 04 / 2020$ & $1: 12$ & No & No & No & Yes & 2 \\
\hline No. 25 & $\begin{array}{l}\text { Decompres- } \\
\text { sion and } \\
\text { arthrodesis } \\
\text { post } 4 \text { levels }\end{array}$ & $11 / 04 / 2020$ & $1: 40$ & Tazocin + Linezolid & No & $\begin{array}{l}\text { Yes, orthope- } \\
\text { dic reason, } \\
2 \text { days }\end{array}$ & Yes & No \\
\hline TOT & & & & 15 & & $\begin{array}{l}3 \text { for ortho- } \\
\text { pedic }\end{array}$ & 24 & 12 \\
\hline
\end{tabular}

outcomes, although all had typical symptoms, especially desaturation requiring oxygen therapy, and in two patients also to CPAP. For this reason, four patients underwent antibiotic therapy, but none underwent a specific therapy for COVID-19. Laboratory data showed a common tendency to lymphopenia. Moreover, all the patients that died had the infection during March 2020.

\section{Discussion}

This study describes the characteristics of suspected and confirmed COVID 19 patients managed at a dedicated Orthopedic and Traumatology facility in Italy. Patients are a consecutive cohort of emergency and trauma patients, managed according to the institutional guidelines produced at the beginning of the pandemic.

\section{Patient's characteristics and comorbidities}

The majority of the positives patients were women. This characteristics may be related to the fact that among the 25 patients there were more women than men. Patients analyzed in this study showed an advanced age and multiple comorbidities. Most of them were affected by fractures. The association of these factors alone may increase the risk of complications and mortality [11]. Considering that COVID19 affected this patient's category, the coexistence of patient characteristics, fracture and infection may have led to an exponential increase in mortality [12]. The most represented comorbidities among COVID-19 patients reported in this study are the same described by the literature: hypertension, cardiovascular diseases, diabetes mellitus, smoking, COPD, malignancy, and chronic kidney disease [13].

\section{Clinical presentation}

There was no difference in symptoms between confirmed positive and suspected CT scan patients. Symptoms reported in this study were similar to those described by the literature [12]. However, fever, cough, and dyspnea are common features in any type of pneumonia, which would explain the high prevalence of interstitial pneumonia on CT scan in an orthopedic ward. As a matter of fact, it should be considered that postoperative pneumonia in patients operated for femoral fracture occurs in about $4.9 \%$ of cases [14], probably due to the inflammatory stress that depresses the immune system [15]. 
Table 9 COVID-19 treatment

\begin{tabular}{|c|c|c|c|c|c|c|c|}
\hline COVID treatment & Oxygen inhalation & Niv & $\operatorname{Imv}$ & Antiviral therapy & Antibacterial therapy & $\begin{array}{l}\text { Hydroxy- } \\
\text { chloroquine } \\
\text { therapy }\end{array}$ & Steroid therapy \\
\hline No. 1 & & & & Oseltamavir & Claritromicina & & \\
\hline No. 2 & Yes & Yes & & & Tazocin + levoxacin & & \\
\hline No. 3 & Yes & Yes & & & & & \\
\hline \multicolumn{8}{|l|}{ No. 4} \\
\hline No. 5 & Yes & Yes & & & & & \\
\hline No. 6 & Yes & Yes & Yes & & & & \\
\hline \multicolumn{8}{|l|}{ No. 7} \\
\hline No. 8 & Yes & Yes & Yes & & & & \\
\hline No. 9 & Yes & Yes & Yes & & & & \\
\hline No. 10 & Yes & Yes & & & & & \\
\hline No. 11 & Yes & Yes & Yes & & & Plaquenil & \\
\hline \multicolumn{8}{|l|}{ No. 12} \\
\hline No. 13 & & & & & Claritromicina & Plaquenil & \\
\hline No. 14 & & & & & & Plaquenil & \\
\hline No. 15 & Yes & Yes & & & & Plaquenil & \\
\hline No. 16 & Yes & Yes & & & $\begin{array}{l}\text { Levoxacin + clari- } \\
\text { tromicina-> Levoxa- } \\
\text { cin + Meropenem }\end{array}$ & & \\
\hline No. 17 & Yes & Yes & Yes & Darunavir & & Plaquenil & Yes \\
\hline No. 18 & Yes & Yes & Yes & Rezolsta & Azitromicina & Plaquenil & \\
\hline No. 19 & Yes & $\begin{array}{l}\text { Yes with } \\
\text { resevoire }\end{array}$ & & & & & \\
\hline No. 20 & Yes & Yes & & & & & \\
\hline No. 21 & Yes & Yes & & & & & \\
\hline No. 22 & Yes & Yes & Yes & & Tazocin + linezolid & & \\
\hline No. 23 & Yes & Yes & & & Tazocin + azitromicina & Plaquenil & \\
\hline No. 24 & Yes & Yes & & & Azitromicina + tazocin & Plaquenil & \\
\hline No. 25 & only in post-op & Yes & & & No & Plaquenil & Yes \\
\hline Total & 19 & 19 & 7 & 3 & 8 & 9 & 2 \\
\hline
\end{tabular}

Considering the 14 femoral fractures, out of the suspected COVID-19-related pneumonia, only two patients had positive swabs, while eight had suspect CT scan; two had false positive CT scan, being affected by COPD exacerbation and cardiopulmonary congestion. Other two suspected patients had not a positive confirmative test; therefore, half of the cases with femoral fractures may have developed a postoperative pneumonia [14].

\section{Predictive role of swab tests and CT scan}

Swab tests performed were positive only in nine patients, while CT scans suspected for COVID-19 pneumonia were 22 (considering whole suspected CT scans, including the simultaneous presence of the positive swab). This discrepancy may be related to a lower swabs test sensitivity compared to the one reported by the literature (about 97\%) [16] or to a tendency to over-diagnose COVID-19 pneumonia utilizing HRCT [17].

Those who reported a history of exposure to COVID-19 patients, or those who were considered as suspected at the ER evaluation, did not necessarily developed a positive swab. The time required to make the diagnosis was quite variable, ranging from one to 13 days, with a delay in the diagnosis greater for patients confirmed by the swab test, although attributable to a longer time needed for the swab's response compared to CT. In 13 patients the diagnosis was suspected or confirmed only after surgical procedure, since a greater exposure to the inflammatory stress intrinsic to operation could depress the immune system, so as to expose subjects to a greater risk of COVID -19 transmission or promoting the development of symptoms in infected patients. 
Table 10 Clinical course data. Deaths are highlighted in Italic

Date of admission Date of discharge Lenght of stay (day) Other compliances during hospi- Clinical outcome

\begin{tabular}{lllcl}
\hline No. 1 & $27 / 02 / 2020$ & $11 / 03 / 2020$ & 13 & Hyperglycemia episode \\
No. 2 & $04 / 03 / 2020$ & $15 / 03 / 2020$ & 11 & No \\
No. 3 & $09 / 03 / 2020$ & $18 / 03 / 2020$ & 9 & Urinary infection \\
No. 4 & $10 / 03 / 2020$ & $28 / 04 / 2020$ & 49 & Stamp necrosis
\end{tabular}

$\begin{array}{lllll}\text { No. } 5 & 14 / 03 / 2020 & 23 / 03 / 2020 & 9 & \text { Other hip dislocation } \\ \text { No. } 6 & 15 / 03 / 2020 & / & 29 & \begin{array}{l}\text { Vertebral fracture L1. Heart failure, } \\ \text { acute renal failure (ARF) on } \\ \text { CKD }\end{array} \\ \text { No. } 7 & 16 / 03 / 2020 & 23 / 03 / 2020 & 7 & \text { No }\end{array}$

No. 8 17/03/2020

$01 / 04 / 2020$

No. $9 \quad 18 / 03 / 2020$

No.10 21/03/2020

$31 / 03 / 2020$

$27 / 03 / 2020$

No. 11 24/03/2020

$01 / 04 / 2020 \quad 8$

No.12 24/03/2020

No.13 25/03/2020

$25 / 03 / 2020$

$24 / 04 / 2020$

No. 14 26/03/2020

$14 / 04 / 2020$

No. 15 28/03/2020

$24 / 04 / 2020$

27

No. 16 28/03/2020

/

10

No. 17 30/03/2020

$23 / 04 / 2020$

No. 18 31/03/2020

$09 / 04 / 2020$

No. 19 31/03/2020

$$
\text { / }
$$

No. 20 31/03/2020

$09 / 04 / 2020$

No. 21 01/04/2020

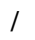

3

No. 22 02/04/2020

I

No. 23 03/04/2020
$20 / 04 / 2020$
17 talization

Stable conditions, transferred to COVID hospital

Stable conditions, transferred to COVID hospital

Stable conditions, transferred to COVID hospital

Good condition, transferred to orthopedic ward cause 2 negative buffer and no symptoms after 14 days of isolation, after discharge at home

Good condition, discharge in another COVID unit

Died on 13 April cause of cardiac arrest

Good condition at discharge, but develops symptoms and is admitted to COVID hospital

Urinary infection (E.Coli)

Good condition, discharge in nursing home

BPCO flare

Episode of desaturation in the emergency room for which he is hospitalized

post-op anemia with ischemic ECG alterations

Good condition, discharge at home

Good condition, isolation at home

No

No

No

No

Silent AMI (cardiac marker positive)

Deep vein thrombosis (DVT) for which caval filter is positioned pre-op

Renal function impairment during ICU and fever

Good condition, discharge in another COVID unit

Good condition, after negative swab discharge at home

Asymptomatic for more 14 days from symptoms, discharge at home

Asymptomatic after spent 14 days of isolation, discharge at home isolation

Asymptomatic after spent 14 days of isolation, transferred to nursing home

Died on 07 April cause of respiratory failure and AMI

Stable conditions, transferred to COVID Hospital

Good condition, discharge in another COVID unit

Phases of AF rhythm, multiple episodes of desaturation

Died on 06 April cause of respiratory distress

AF rhythm

No

Good condition, discharge in nursing home

Died on 04 April cause of cardiac arrest

Multiple atrial fibrillation episode, psychomotor agitation and multiorgan worsening

No
Died on 23 April cause of psychophysical decay

Asymptomatic after spent 14 days of isolation, discharge at home 
Table 10 (continued)

Date of admission Date of discharge Lenght of stay (day) Other compliances during hospi- Clinical outcome talization

\begin{tabular}{|c|c|c|c|c|c|}
\hline No. 24 & 04/04/2020 & $20 / 04 / 2020$ & 16 & No & $\begin{array}{l}\text { Asymptomatic after spent } 14 \text { days of } \\
\text { isolation, discharge at home }\end{array}$ \\
\hline No. 25 & $11 / 04 / 2020$ & I & in progress & & Stable conditions \\
\hline TOT & & & & 16 & Five died \\
\hline
\end{tabular}

\section{Main laboratory findings}

Laboratory data analysis showed that COVID-19 patients had anemia, leukocytosis, neutrophilia, lymphopenia, and thrombocytosis. Furthermore, sporadic alterations in coagulation and in liver and kidney function have been observed. However, anemia is a common condition in surgically treated orthopedic patients, and alterations of the leukocyte formula were reported even before surgical procedure and in correspondence to the symptoms in three patients.

Many studies converge on the uniqueness of the laboratory data, also proposing to utilizing blood results as a diagnostic support for rt-PCR, observing recurrent alterations in positive patients [18], including WBC, CRP, AST, and ALT [19]. In our study, all the nine positive swabs patients showed with alterations such as those reported in the other studies. Moreover, swabs positive patients showed alterations in LY, PLT, and ALP, especially lymphopenia recurred in seven of nine patients with positive swab test, while it was found in six of the 16 suspected cases. Zeng et al. took these parameters into account stating that severe COVID-19 patients had more neutrophils and fewer lymphocytes cells [20].

\section{Patient's management and ICU access}

Elderly patients with hip fractures and multiple comorbidities take advantage of being subjected to surgery as soon as possible, within $48 \mathrm{~h}$ : this allows for early mobilization, reduced bed rest, better pain control, and reduced complications including deep vein thrombosis, pneumonia, and overall mortality $[21,22]$. In this cohort of consecutive trauma patients, surgical procedure was performed in hemodynamically stable subjects, with an oxygen saturation higher than $90 \%$. The performance of this selection, as suggested by some recent studies [23, 24], may have contributed to obtain a favorable outcome, comparable to non-COVID patients.

The institutional protocol of anti-thromboembolic prophylaxis, administered to all the orthopedic patients at the authors' institution could have contributed to the reduction of mortality rates and complications of the COVID-19 infection, as suggested by a recent study [25].

Considering the different therapy patterns utilized among the 25 patients, there were no significant differences on the time taken for symptom regression (ranging from three to 14 days) or on the negative turn of the swab (more than 14 days). In particular, therapy with hydroxychloroquine or azithromycin did not show advantages over other drugs or pharmacological therapeutic abstention, in agreement with the most recent randomized studies [26, 27].

\section{Clinical course and outcomes}

Three patients had delayed surgical procedure (beyond three days from admission) because of respiratory symptoms onset. Out of these, two died at first and 25th days after surgical procedure, respectively. The other three deaths occurred at first, seventh, and 20th days from surgical procedure. This variability does not allow to establish whether surgical procedure was significant to influence the prognosis, but according to the results of this study (Table 5), surgical procedure may represent a risk factor for COVID-19 infection to become symptomatic, by depressing the immune system [15]. Deceased patients had several risk factors of poor outcome including age, type of fracture, and multiple comorbidities [12]. Moreover, since all the five patients had negative test for swabs and the diagnosis of COVID-19 was performed on the evidence of interstitial-alveolar pneumonia by HRCT, doubts may arise on the role of COVID-19 infection.

The number of discharges reported in this study is greater than $52 \%$ compared to the literature. On the other hand, the number of deaths reach $20 \%$, compared to $5 \%$ of the international studies [28].

In the current report, 18 of the 25 patients developed a presumed nosocomial COVID-19 infection (Fig. 2). A possible risk factor may have been the initial unpreparedness of the staff in patient's management, denounced by the fact that most of the cases date back to the month of March (first period). In these patients, the long time required to make the diagnosis, which in 13 cases was carried out after surgical procedure, may have played a role.

Strengths of this study are represented by the consecutive patient's enrollment, of which a complete set of data is retrieved by the medical charts. Main limitations are the relatively small group of patients, the absence of control group, the heterogeneity of comorbidities, and the lack of a standard for pharmacology treatment. 


\section{Conclusions}

This present paper reports the clinical and laboratory characteristics of suspected and diagnosed consecutive COVID 19 patients managed at a single institution during the first pandemic period. This present study may contribute to the ability of doctors in approaching such patients. The development of a patient management algorithms allows the differentiation of the clinical pathways of negative and suspected/ positive patients, reducing exposure, and virus spreading. Patient management protocols implemented beginning late April allowed an earlier diagnosis, since the swabs were performed to all new admitted patients. Further research is required to optimize treatment strategies, establish shared protocols and gain a better understanding on COVID-19 patient's characteristics and possible risk factors related to trauma surgery.

Acknowledgements The authors acknowledge all the nurses and health care staff who took care of patients during this dramatic period.

Funding No funding was received for this study.

Data availability Code availability Not applicable.

\section{Declarations}

Conflict of interest The authors declare that they have no conflict of interest.

Ethical approval This article does not contain any studies with human participants or animals performed by any of the authors.

Consent to participate and publication All the patients provided their informed consent at admission on data collection and reporting.

\section{References:}

1. Cucinotta D, Vanelli M (2020) WHO declares COVID-19 a pandemic. Acta Bio-Medica Atenei Parm 91:157-160. https://doi.org/ 10.23750/abm.v91i1.9397

2. Paterlini $M(2020)$ On the front lines of coronavirus: the Italian response to covid-19. BMJ 368:m1065. https://doi.org/10.1136/ bmj.m1065

3. Zagra L, Faraldi M, Pregliasco F et al (2020) Changes of clinical activities in an orthopaedic institute in North Italy during the spread of COVID-19 pandemic: a seven-week observational analysis. Int Orthop. https://doi.org/10.1007/s00264-020-04590-1

4. Grassi A, Pizza N, Tedesco D, Zaffagnini S (2020) The COVID-19 outbreak in Italy: perspectives from an orthopaedic hospital. Int Orthop. https://doi.org/10.1007/s00264-020-04617-7

5. Leti Acciaro A, Montanari S, Venturelli M et al (2021) Retrospective study in clinical governance and financing system impacts of the COVID-19 pandemic in the hand surgery and microsurgery HUB center. Musculoskelet Surg. https://doi.org/10.1007/ s12306-021-00700-3
6. Faldini C (2020) Reorganization of the Rizzoli Orthopaedic Institute during the COVID-19 outbreak. Musculoskelet Surg 104:227-228. https://doi.org/10.1007/s12306-020-00688-2

7. Chughtai M, Gwam CU, Mohamed N et al (2017) The Epidemiology and Risk Factors for Postoperative Pneumonia. J Clin Med Res 9:466-475. https://doi.org/10.14740/jocmr3002w

8. Abdelmalek A, Crowther M (2021) Olecranon fractures in the elderly during the COVID-19 pandemic: Is non-operative treatment reasonable? Review of the current evidence. Musculoskelet Surg. https://doi.org/10.1007/s12306-021-00699-7

9. Rothe C, Schunk M, Sothmann P et al (2020) Transmission of 2019-nCoV infection from an asymptomatic contact in Germany. N Engl J Med 382:970-971. https://doi.org/10.1056/NEJMc20014 68

10. Chen Z-Y, Wu H-Z, Zhu P, Feng X-B (2015) Postoperative changes in hemoglobin and hematocrit in patients undergoing primary total hip and knee arthroplasty. Chin Med J (Engl) 128:1977-1979. https://doi.org/10.4103/0366-6999.160620

11. Lizaur-Utrilla A, Lopez-Prats FA (2020) Hip attack for hip fractures: is ultra-early surgery necessary? Lancet Lond Engl 395:661-662. https://doi.org/10.1016/S0140-6736(20)30156-2

12. Zhu Y, Chen W, Xin X et al (2020) Epidemiologic characteristics of traumatic fractures in elderly patients during the outbreak of coronavirus disease 2019 in China. Int Orthop. https://doi.org/10. 1007/s00264-020-04575-0

13. Emami A, Javanmardi F, Pirbonyeh N, Akbari A (2020) Prevalence of underlying diseases in hospitalized patients with COVID19: a systematic review and meta-analysis. Arch Acad Emerg Med 8:e35

14. Lv H, Yin P, Long A et al (2016) Clinical characteristics and risk factors of postoperative pneumonia after hip fracture surgery: a prospective cohort study. Osteoporos Int J Establ Result Coop Eur Found Osteoporos Natl Osteoporos Found USA 27:3001-3009. https://doi.org/10.1007/s00198-016-3624-5

15. Zhang P, Xia G, Dai L et al (2019) Laryngoscope-assisted and cotton ball wiping methods in prevention of oral and pulmonary infection in patients receiving mechanical ventilation and the influence on hypersensitive C-reactive protein and procalcitonin. Exp Ther Med 18:531-536. https://doi.org/10.3892/etm.2019. 7614

16. Castro R, Luz PM, Wakimoto MD et al (2020) COVID-19: a meta-analysis of diagnostic test accuracy of commercial assays registered in Brazil. Braz J Infect Dis Off Publ Braz Soc Infect Dis 24:180-187. https://doi.org/10.1016/j.bjid.2020.04.003

17. Hernigou J, Cornil F, Poignard A et al (2020) Thoracic computerised tomography scans in one hundred eighteen orthopaedic patients during the COVID-19 pandemic: identification of chest lesions; added values; help in managing patients; burden on the computerised tomography scan department. Int Orthop. https:// doi.org/10.1007/s00264-020-04651-5

18. Ferrari D, Motta A, Strollo M et al (2020) Routine blood tests as a potential diagnostic tool for COVID-19. Clin Chem Lab Med. https://doi.org/10.1515/cclm-2020-0398

19. R, Ahmadi Vasmehjani A, Zali F, et al (2020) Laboratory Parameters in Detection of COVID-19 Patients with Positive RT-PCR; a Diagnostic Accuracy Study. Arch Acad Emerg Med 8:e43

20. Zeng F, Li L, Zeng J, et al (2020) Can we predict the severity of coronavirus disease 2019 with a routine blood test? Pol Arch Intern Med 130:400-406. https://doi.org/10.20452/pamw.15331

21. Ravi B, Pincus D, Wasserstein D et al (2018) Association of overlapping surgery with increased risk for complications following hip surgery: a population-based, matched Cohort Study. JAMA Intern Med 178:75-83. https://doi.org/10.1001/jamainternmed. 2017.6835

22. Merloz P (2018) Optimization of perioperative management of proximal femoral fracture in the elderly. Orthop Traumatol Surg 
Res OTSR 104:S25-S30. https://doi.org/10.1016/j.otsr.2017.04. 020

23. Mi B, Chen L, Xiong Y et al (2020) Characteristics and Early Prognosis of COVID-19 Infection in Fracture Patients. J Bone Joint Surg Am 102:750-758. https://doi.org/10.2106/JBJS.20. 00390

24. Catellani F, Coscione A, D'Ambrosi R et al (2020) Treatment of proximal femoral fragility fractures in patients with COVID-19 during the SARS-CoV-2 outbreak in Northern Italy. J Bone Joint Surg Am. https://doi.org/10.2106/JBJS.20.00617

25. Tang N, Bai H, Chen X et al (2020) Anticoagulant treatment is associated with decreased mortality in severe coronavirus disease 2019 patients with coagulopathy. J Thromb Haemost JTH 18:1094-1099. https://doi.org/10.1111/jth.14817

26. Rosenberg ES, Dufort EM, Udo T et al (2020) Association of treatment with hydroxychloroquine or azithromycin with in-hospital mortality in patients with COVID-19 in New York State. JAMA. https://doi.org/10.1001/jama.2020.8630

27. Mahévas M, Tran V-T, Roumier M et al (2020) Clinical efficacy of hydroxychloroquine in patients with covid-19 pneumonia who require oxygen: observational comparative study using routine care data. BMJ 369:m1844. https://doi.org/10.1136/bmj.m1844

28. Li L-Q, Huang T, Wang Y-Q et al (2020) COVID-19 patients' clinical characteristics, discharge rate, and fatality rate of metaanalysis. J Med Virol. https://doi.org/10.1002/jmv.25757

Publisher's Note Springer Nature remains neutral with regard to jurisdictional claims in published maps and institutional affiliations. 